\title{
Utilização de pesquisas: Como construir modelos teóricos para avaliação?
}

\author{
Research use: how to build a theoretical \\ model of evaluation?
}

Flávia Tavares Silva Elias 1

Maria Aparecida de Assis Patroclo 2

\footnotetext{
${ }^{1}$ Departamento de Ciência Tecnologia/SCTIE/ Ministério da Saúde. Condomínio Verde, Rua Buritis 13, SHIS, 71680-608, Brasília DF. flavia.elias@saude.gov.br 2 Secretaria Municipal de Saúde do Rio de Janeiro, Investigação de Sistemas e Serviços de Saúde, OPAS/ENSP/Fiocruz.
}

\begin{abstract}
Many international forums agree that scientific investigations are a subsidy for the decision making process in health. The goal of this article is that of making a bibliographical revision which may allow the proposal of a suitable model for evaluating how results of researches are used in the development of health policies. Two authors were chosen as a theoretical referential due to their contributions in the contexts of America (Weiss, C.) and Latin-America (Trostle et al.). The following components were included in the theoretical model: research, interaction, communication, players, social pressure and funding. Results were seen according to a specific criteria as to better evaluate each component. A matrix to evaluate how efficient the use of research data may be was developed considering categories as follows: structural, operational, strategic, systemic and specific. For demonstrate the balance of forces involved in the process of linking research to policies, an encouragement-obstacle matrix was developed. Social, economic, political, cultural and managerial dimensions were considered. The set including the model and the matrixes subsidize to select the indicators for evaluating and monitoring any program that may base itself on results of researches of the Ministry of Health.
\end{abstract}

Key words Theoretical model of evaluation, Indicators of the the use of research
Resumo Diversos fóruns internacionais consideram que investigações podem subsidiar a tomada de decisão em saúde. O artigo tem como objetivo a revisão bibliográfica para propor modelo de avaliação do uso de resultados de pesquisas em politicas de saúde. Dois autores foram selecionados como referencial teórico, pela contribuição no contexto americano (Weiss, C.) e latino-americano (Trostle et al.). Foram selecionados os seguintes componentes como parte do modelo teórico: pesquisa, comunicação, interação, atores, pressão social e financiamento. Nos resultados incluíramse critérios para avaliar cada componente. Elaborou-se matriz de efetividade da utilização de pesquisa com as categorias de análise: estrutural, operacional, estratégica, sistêmica e específica. Para demonstrar o balanço de forças atuantes no processo pesquisa/politica elaborou-se matriz de fatores de promoção e de impedimento. As dimensões sociais, econômica, política, cultural e gerencial foram selecionadas. O conjunto composto pelo modelo teórico e as matrizes subsidiam a seleção de indicadores de monitoramento e avaliação de um programa de utilização de resultados de pesquisas do Ministério da Saúde.

Palavras-chave Modelo teórico de avaliação, Indicadores do uso de pesquisa 


\section{Introdução}

No contexto internacional, diversos fóruns mundiais e latino-americanos consideram que o uso de informações geradas por investigações científicas pode subsidiar a alocação de recursos, visando ao maior benefício à saúde da população e integrando uma estratégia global de desenvolvimento social com eqüidade (WHO/ ACHR, 1998, WHO, 1998). Destacam-se as recomendações do Council on Health Research for Development (COHRED), que constituiu um grupo de trabalho para aprimorar as estratégias de integração entre pesquisa e ação e, em particular, pesquisa e política (COHRED, 2000a; OPS, 2000)

$\mathrm{Na}$ América Latina e Caribe, fórum regional promovido, em 2000, pela Organização Pan-Americana da Saúde (OPS), intitulado Usos da Investigação em Reformas do Setor Saúde, foi um marco no sentido de intensificar o subcampo de investigações em sistemas e serviços de saúde (OPS, 2000).

Em 2001, na reunião preparatória da Cúpula das Américas, promovida pela OPS, assumiu-se que o processo de produção, tradução, utilização e disseminação de conhecimento é complexo e exige responsabilidade tripartite de pesquisadores, gestores e usuários de serviços.

No Brasil, o Sistema Único de Saúde (SUS) tem atribuições voltadas para incrementar o desenvolvimento científico e tecnológico em saúde e a avaliação do impacto que as tecnologias provocam à saúde (lei 8.080/90). A 1ạ Conferência de Ciência e Tecnologia em Saúde (MS, 1994) foi um marco para promoção de articulações intersetoriais no sentido de elaborar diretrizes para definição de uma política de ciência e tecnologia em saúde.

Ao Ministério da Saúde (MS), gestor federal do SUS, compete também formular, avaliar, elaborar normas e participar na execução da política nacional de produção de insumos e equipamentos para a saúde. Para cumprir com as atribuições, em 2001, o Ministério estruturou o Departamento de Ciência e Tecnologia (DECIT) e, em 2003, foi criada a Secretaria de Ciência, Tecnologia e Insumos Estratégicos. No Plano Plurianual estão orçados para 2004 cerca de 260 milhões no âmbito do programa de ciência, tecnologia e inovação em saúde. Desta forma, o Ministério da Saúde é financiador, usuário e indutor de pesquisa em saúde.

Existem vários estudiosos no campo da utilização de resultados de pesquisas, tais como:
D. Donnison (1972), Weiss C. (1975, 1978, 1979, 1991); Nathan C. (1975); Weiss J. (1976); Wittrock B. (1991); Frenk J. (1992); Barthley M. (1992); Pahl J. (1992); Hailey D. M. (1993); Ferguson J. H. (1993); Mckinlay J. (1993); Haines A. (1994); Walt G. (1994); Stocking B. (1995); Porter R. W. (1995); Davis P. et al. (1996); Brownson R. C. et al. (1997, Trostle J. et al. (1999). Neste estudo, entretanto, serão considerados dois teóricos, selecionados por serem referências e por representarem contextos culturais distintos: contexto cultural norteamericano (Weiss C.) e contexto cultura latino-americano (Trostle J.).

Considerando os múltiplos caminhos possíveis para a elaboração de modelos teóricos e construção de indicadores de avaliação da utilização de pesquisas, iremos, a partir da descrição e análise de conceitos, destacar questões a serem consideradas em qualquer avaliação dessa natureza.

Na década de 1970, nos Estados Unidos da América do Norte, estudos realizados por Weiss (1979) referiam que os cientistas sociais estavam preocupados sobre como fazer uso de suas pesquisas para formulação de políticas públicas e que os gestores manifestavam preocupação com a utilidade das pesquisas financiadas por órgãos governamentais. Havia interesse mútuo em saber se as pesquisas em ciências sociais que pretendiam influenciar políticas eram de fato "usadas"; mas era necessário o entendimento do que significava "utilização de pesquisa".

A autora fez uma revisão na literatura, revelando diferentes significados para o termo "utilização de pesquisa", analisou como a pesquisa tem afetado e podia influenciar políticas, e apresentou sete modelos associados ao significado do conceito.

Na figura 1, representamos graficamente os modelos elaborados por Weiss (1979) e identificamos questões relevantes a serem consideradas em modelos teóricos de avaliação do uso de pesquisas em políticas de saúde.

1) Modelo do conhecimento dirigido: é o mais descrito na literatura e deriva das ciências naturais. Assume seqüência linear e o fato de o conhecimento existir pressiona o seu desenvolvimento e uso (Figura 1).

As questões a serem consideradas nesse modelo do conhecimento dirigido são: a) Os resultados de pesquisa permitem o desenvolvimento de novas tecnologias? b) O estágio em que se encontra o conhecimento permite que as novas tecnologias sejam desenvolvidas em curto pra- 
Modelo de resolução de problemas

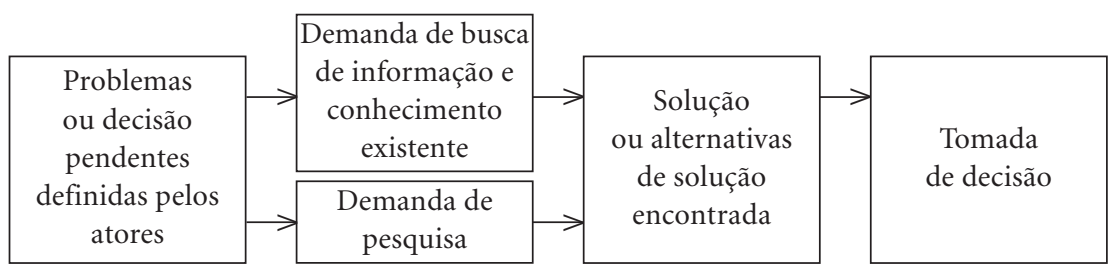

\section{Modelo interativo}

\begin{tabular}{|c|c|c|c|}
\hline $\begin{array}{l}\text { Decisão } \\
\text { pendente }\end{array}$ & $\begin{array}{c}\text { Busca de } \\
\text { informação e } \\
\text { conhecimento } \\
\text { em várias fontes }\end{array}$ & $\begin{array}{c}\text { Conexões entre } \\
\text { experiência, } \\
\text { pesquisa } \\
\text { produzida, mídia, } \\
\text { etc. estabelecidas }\end{array}$ & $\begin{array}{c}\text { Tomada } \\
\text { de decisão }\end{array}$ \\
\hline
\end{tabular}

\section{Modelo político}

\begin{tabular}{|c|c|c|c|c|}
\hline $\begin{array}{l}\text { Obtenção e } \\
\text { utilização de } \\
\text { resultados de } \\
\text { pesquisa por } \\
\text { ideologia ou } \\
\text { inteligência }\end{array}$ & $\begin{array}{c}\text { Neutralização } \\
\text { de oponentes, } \\
\text { convecimento } \\
\text { de indecisos, } \\
\text { sustentção de } \\
\text { partidários }\end{array}$ & $\begin{array}{c}\text { Acesso a } \\
\text { evidências } \\
\text { científicas, } \\
\text { a todos os } \\
\text { participantes do } \\
\text { processo político }\end{array}$ & $\begin{array}{l}\text { Advocacia, } \\
\text { redução de } \\
\text { incertezas, } \\
\text { finalização de } \\
\text { debates }\end{array}$ & $\begin{array}{l}\text { Tomada } \\
\text { de decisão }\end{array}$ \\
\hline
\end{tabular}

\section{Modelo tático}

\begin{tabular}{|c|c|c|c|c|c|}
\hline Pressão social & $\begin{array}{l}\text { Pesquisa como } \\
\text { recurso tático }\end{array}$ & $\begin{array}{c}\text { Promessa de ação } \\
\text { a partir dos } \\
\text { resultados de } \\
\text { pesquisa }\end{array}$ & $\begin{array}{c}\text { Utilização de } \\
\text { agências ou } \\
\text { pesquisadores de } \\
\text { prestígio }\end{array}$ & $\left\{\begin{array}{c}\text { Tentativa de evitar } \\
\text { responsabilização } \\
\text { direta por política } \\
\text { impopular }\end{array}\right.$ & $\begin{array}{l}\text { Legitimação } \\
\text { de política } \\
\text { burocrática }\end{array}$ \\
\hline
\end{tabular}

\section{Modelo iluminador/esclarecedor}

\begin{tabular}{|c|c|c|c|}
\hline $\begin{array}{c}\text { Resultados de } \\
\text { pesquisa divulgados } \\
\text { canais em formais } \\
\text { e informais }\end{array}$ & $\rightarrow \begin{array}{c}\text { Suportes } \\
\text { adequados/ } \\
\text { inadequados }\end{array}$
\end{tabular}$\rightarrow \begin{gathered}\text { Decisões } \\
\text { Mudanças de } \\
\text { parâmetros } \\
\text { sensatas/ } \\
\text { insensatas }\end{gathered}$

\section{Modelo empreendedor}

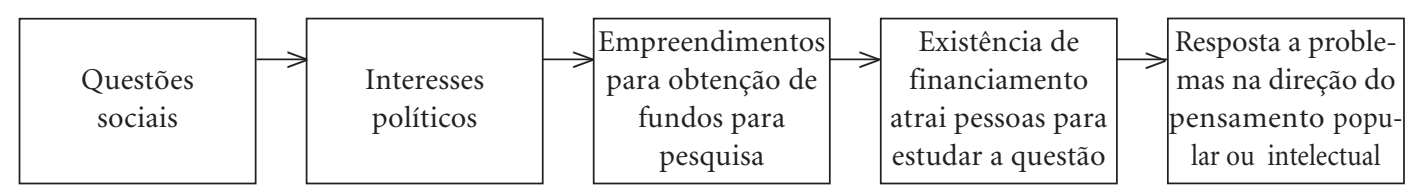


zo? c) As novas tecnologias teriam menor ou maior custo? Trariam benefício, efetividade e segurança igual ou maior do que as já existentes?

2) Modelo de resolução de problema: a concepção mais comum de utilização de pesquisa, envolve a aplicação direta de resultados de um estudo específico para a solução de um problema ou para a tomada de decisão pendente. A expectativa é que a pesquisa forneça evidências empíricas e conclusões para ajudar a resolver um problema. A utilização dos resultados da pesquisa só é possível se os atores principais formuladores de política - definem com precisão o problema; se os resultados da pesquisa não são ambíguos, se possuem bases teóricas sólida e não são contrários a fortes interesses políticos e se apontam para uma solução ou alternativas de solução que permitam a tomada de decisão (Figura 1).

As questões a serem consideradas no modelo resolução de problemas são: a) Os formuladores de política solicitaram pesquisas para resolver problemas ou tomar decisão? b) $\mathrm{O}$ problema ou decisão pendente estava bem definido? d) Os resultados de pesquisa forneceram alternativas de solução? Contrariam fortes interesses políticos?

3) Modelo interativo: os formuladores de política buscam a informação em diferentes fontes e consultam diferentes grupos sociais. São utilizadas experiências acumuladas, pressão social, resultado de pesquisa, entre outros insumos, e assim se estabelecem conexões para tomada de decisão. O pesquisador faz parte de um dos grupos de informantes e a pesquisa pode ser rejeitada, usada parcial ou totalmente, não estando necessariamente representada na política adotada (Figura 1).

As questões a serem consideradas no modelo interativo são: a) Foram consideradas experiências e informações de vários grupos sociais para formulação da política? b) Os resultados de pesquisa ou opinião de pesquisadores foram incluídos como categoria mais importante na formulação da política?

4) Modelo político: os formuladores de política utilizam a pesquisa por interesse ideológico ou por inteligência para convencer indecisos, neutralizar oponente, sustentar partidários. Há acesso das evidências científicas sem distorções aos participantes do processo decisório e por meio da advocacia há redução de incertezas e finalização de debates. É o uso estratégico da pesquisa no processo decisório. O resultado da pesquisa não estará presente na política final (Figura 1).
As questões a serem consideradas no modelo político são: a) Os governantes/formuladores de políticas utilizaram resultados de pesquisa com que finalidade? b) Os participantes tiveram acesso às evidências científicas sem distorções?

5) Modelo tático: a partir de demandas sociais por ação, os formuladores de política utilizam a necessidade de pesquisa como recurso tático para conter pressões e prometem ação a partir do resultado de pesquisa. Serve para evitar a responsabilização direta se tiverem de implantar uma política impopular (Figura 1).

As questões a serem consideradas no modelo tático são: a) Existia pressão social por ação governamental para resolver o problema? b) Houve promessa dos governantes de agirem a partir de resultados de pesquisa? c) Houve encomenda de pesquisa por parte dos governantes dirigida apenas a agências e pesquisadores com prestígio? d) Houve implantação de política impopular baseada ou justificada nos resultados de pesquisa?

6) Modelo iluminador/esclarecedor: a difusão de resultados da pesquisa se dá por canais formais e informais que têm como possíveis conseqüências a conversão de problemas em não problemas e vice-versa. Gera a mudança de parâmetros sociais baseados em suporte que pode iluminar ou obscurecer a tomada de decisão (Figura 1).

As questões a serem consideradas no modelo iluminador são: a) Os resultados de pesquisas eram contraditórios com os parâmetros da sociedade civil? Tiveram divulgação por canais de comunicação formais e informais? b) Os resultados de pesquisa podem trazer mudanças de parâmetros, que serão nefastos para um grupo maior do que os que deles podem se beneficiar? 7) Modelo empreendedor: a política e a pesquisa respondem a correntes de pensamento, modas passageiras e fantasias do período; interagem influenciando uma a outra e são influenciadas pelo pensamento social. Interesses políticos emergem para questões sociais, que conduzem a empreendimentos para obtenção de fundos para pesquisa e somente com a disponibilidade dos fundos os pesquisadores são atraídos para estudar a questão. Ambos, política e pesquisa, podem responder consciente ou inconscientemente a problemas que se movem com rapidez em direção ao pensamento popular ou intelectual. A pesquisa é parte interconectada do empreendimento intelectual (Figura 1).

As questões a serem consideradas no modelo empreendedor são: a) A principal motiva- 
ção dos pesquisadores foi o financiamento para o estudo do problema? b) As pesquisas consideraram apenas os aspectos oficiais de interesse dos governantes/formuladores de política? c) Os governantes empreenderam esforços para captarem fundos para as pesquisa? d) As políticas tiveram direcionamento para o pensamento popular ou intelectual?

No contexto latino-americano, estudo realizado no México por Trostle et al. (1999) analisa os modelos sugeridos por Weiss (1979) e os sintetiza em três abordagens. A primeira, denominada racional, reúne o modelo do conhecimento dirigido e de resolução de problema e representa o pensamento tradicional de utilização de pesquisa, no qual os acontecimentos possuem seqüência linear. O processo político é racional e os formuladores de política usarão os resultados de pesquisa se estas existirem. A segunda é a estratégica, que agrega o modelo político e tático, em que a pesquisa é útil para apoiar posições predeterminadas ou validar decisões. A terceira é a esclarecedora ou difusora; agrega os modelos interativo, esclarecedor e empreendedor, nos quais a pesquisa e a tomada de decisão influenciam uma a outra, e são influenciadas pelo processo social.

Para Trostle et al. embora esses modelos esclareçam as razões do uso de pesquisas em políticas, eles esclarecem muito pouco sobre o processo de formulação de políticas e são bastante abstratos para facilitarem a comparação entre pesquisa e política. A partir dessas abordagens e da perspectiva de que o processo contingencia o desenvolvimento e implementação de mudanças no contexto em que a política é desenvolvida, Trostle et al. consideram relevantes as categorias: conteúdo, atores, processo e contexto para compreensão da contribuição específica da pesquisa na formulação de políticas.

Torna-se necessário elaborar mapas de atores e contexto envolvendo pesquisa e política. O Estado e a sociedade civil têm áreas de intersecção. Vários grupos de interesse agem para influenciar políticas. Os pesquisadores são somente um entre esses muitos grupos. Grupos de interesse e formuladores de política exercem influências mútuas. Alguns grupos de interesse como a igreja ou a indústria privada pertence exclusivamente à sociedade civil. Outros, como ministro da Saúde e legisladores, pertencem ao Estado, assim como pesquisadores de institutos estatais pertencem a ambos.

Os processos de pesquisa e de política são dinâmicos. Ocorrem usualmente em lugares independentes, mas podem caminhar juntos em vários momentos. Esses possíveis contatos entre os dois processos são oportunos para que os participantes contribuam um com o outro. A aplicação de pesquisa nas políticas consiste em criar ou reconhecer os momentos de oportunidade, e então agir eficientemente para obter vantagens deles.

O processo de pesquisa inclui as fases de geração de idéias, desenho do estudo, coleta de dados, análise e aplicação dos resultados. Os resultados de pesquisa criam novas idéias e novos projetos de pesquisa; um caminho retorna para os estágios iniciais enquanto outro segue para a aplicação dos resultados da pesquisa. A aplicação dos resultados também gera novas idéias e novos desenhos.

O processo de formulação de políticas se inicia quando necessidades ou problemas surgem e devem ser resolvidos através de políticas. As informações sobre essas necessidades e problemas são coletadas em diferentes fontes. Grupos de interesse exercem pressão em vários estágios: na definição do tipo de necessidades que serão reconhecidas e as que serão ignoradas; no tipo de decisões que serão tomadas e no tipo de políticas que emergirão. Como no processo de pesquisa, alguns caminhos conduzem a um retorno para busca de informações antes das políticas serem formuladas. Algumas decisões ocasionam a procura de informações adicionais e novas negociações, enquanto outras decisões geram políticas. Novas políticas criam novos grupos de interesse e novas mudanças políticas.

Com base no referencial anteriormente citado, os autores (Trostle et al.), visando reconstruir o processo pelo qual pesquisas foram usadas para tomada de decisão e formulação de políticas, elegeram para avaliação quatro programas verticais (imunização, DST/Aids, cólera e planejamento familiar) do Ministério da Saúde do México, em que havia necessidade de revisão de normas. Nesses programas existiam algumas interações entre pesquisa e política.

As opiniões de atores entrevistados demonstraram a existência de fatores de promoção e de impedimento para utilização de resultados de pesquisa. Esses fatores foram categorizados em conteúdo, atores, processo e contexto na interação pesquisa-política.

1) Conteúdo de pesquisa e de políticas (Quadro 1): envolve características estruturais de pesquisa e de política, ou seja, a abrangência e urgência do tema, inovação, complexidade, qualidade, reprodutilibidade, inteligibilidade, 
custo, benefício, beneficiários, requisitos técnicos e nível de participação popular necessário para implementação.

As questões a serem consideradas indicadores/critérios para avaliação de fatores de promoção e de impedimento em relação ao conteúdo são: a) $\mathrm{O}$ tema de pesquisa foi definido como prioridade governamental? b) Os resultados de pesquisas tiveram recomendações aplicáveis? Foram divulgados em revistas e jornais de grande circulação ou Internet? c) O pesquisador titular tinha fama e prestígio na comunidade científica e/ou no governo?

2) Quanto aos atores (Quadro 2): são indivíduos ou grupos que atuam na criação ou obstrução de políticas, caracterizados pela sua identidade (quem está envolvido no processo de formulação de política e de pesquisa) e pela sua qualidade (nível de motivação, liderança, treinamento, acesso ao poder, prestígio). Para identificá-los é necessário conhecer que infor- mações cada um considera relevante, quais interessam a cada um deles, como avaliam a informação, que motivação possuem para tomar decisões e com quem interagem.

As questões a serem consideradas indicadores/critérios para avaliação de fatores de promoção e de impedimento em relação aos atores são: a) Houve participação e consenso dos governantes/formuladores de políticas na definição de prioridades de pesquisa? b) A pesquisa teve financiamento de agências governamentais de saúde ou organizações internacionais? c) O formulador de política percebia as informações científicas como importantes para tomada de decisão? Os pesquisadores fizeram recomendações capazes de serem compreendidas pelos formuladores de políticas?

3) Processos de formulação política e de pesquisa (Quadro 3): as variáveis de processo são os tipos de canais de comunicação, como eles são usados, como os resultados de pesquisa se

Quadro 1

Fatores de promoção e de impedimento relativos ao conteúdo de pesquisa e de política.

\begin{tabular}{ll}
\hline Fatores de promoção & Fatores de impedimento \\
\hline $\begin{array}{l}\text { Qualidade da pesquisa - mensuração: Prestígio do } \\
\text { pesquisador, boa relação pesquisador e formulador } \\
\text { de políticas, reputação de jornais e revistas nos } \\
\text { quais os resultados de pesquisa são publicados }\end{array}$ & $\begin{array}{l}\text { Diferenças entre vocabulários de pesquisadores } \\
\text { e de formuladores de política }\end{array}$ \\
$\begin{array}{l}\text { Maior atenção para os resultados de pesquisas } \\
\text { biomédicas }\end{array}$ & $\begin{array}{l}\text { Diferentes vocabulários em diferentes tipos } \\
\text { de pesquisa }\end{array}$ \\
$\begin{array}{l}\text { Especificidade, concretude e custo efetividade } \\
\text { dos resultados de pesquisa }\end{array}$ & $\begin{array}{l}\text { dos formuladores de políticas da utilidade } \\
\text { de pesquisas e falta de percepção dos pesquisadores } \\
\text { sobre características das recomendações para } \\
\text { influenciarem as políticas }\end{array}$ \\
\hline
\end{tabular}

Quadro 2

Fatores de promoção e de impedimento relativos aos atores envolvidos com a pesquisa e a política.

\begin{tabular}{ll}
\hline Fatores de promoção & Fatores de impedimento \\
\hline $\begin{array}{l}\text { Participação conjunta de governantes/ } \\
\text { formuladores de políticas e pesquisadores } \\
\text { na definição de problemas prioritários }\end{array}$ & $\begin{array}{l}\text { Baixo background técnico dos formuladores } \\
\text { de política e da mídia }\end{array}$ \\
Suporte financeiro internacional & $\begin{array}{l}\text { Cultura política: decisões baseadas na experiência } \\
\text { e em pressões imediatas, falta de percepção } \\
\text { de como usar resultados de pesquisa }\end{array}$ \\
Pressão exercida por organismos internacionais & $\begin{array}{l}\text { Agenda política influenciada por interesses } \\
\text { de grupos, especialmente os financeiros }\end{array}$ \\
\hline
\end{tabular}


movem nas organizações, que oportunidades surgiram para uso de pesquisa, que tipo de eventos inesperados promovem ou impedem o uso de pesquisa em políticas.

As questões a serem consideradas indicadores/critérios para avaliação de facilidades e de impedimento em relação ao processo são: a) Que relações existem entre pesquisadores e formuladores de política? b) Existem canais de comunicação para disseminação de resultados de pesquisas? c) Qual a correlação de forças entre os grupos envolvidos no processo de formulação de políticas?

4) Contexto político-institucional (Quadro 4): refere-se aos cenários políticos e institucionais em que ocorre a construção da pesquisa e da política.

As questões a serem consideradas indicadores/critérios para avaliação de facilidades e de impedimento em relação ao contexto são: a) $\mathrm{O}$ problema de saúde pesquisado era urgente? Havia estabilidade política no governo? Houve consenso e coesão da comunidade científica em relação ao tema pesquisado? b) O governante centraliza as informações? Houve restrições financeiras para o tema a ser objeto da política?

Considerando os autores analisados, observamos que resultados de pesquisa podem ser aplicados e para Weiss o impacto específico de um estudo específico para uma decisão específica é somente um indicador do uso de resultados de pesquisa, nós teremos de inventar modos mais complexos e mais apropriados para avaliar esse uso.

Este artigo, com base no desafio dos autores, inova ao propor, a partir da revisão bibliográfica, modelo teórico e matrizes de indicadores para avaliação da viabilidade de programa hipotético de utilização de resultados de pesquisas, financiadas por órgãos governamentais brasileiros, na formulação de políticas de saúde.

\section{Metodologia}

Adotamos os modelos de utilização de pesquisa abordados por Weiss (1979) para definir e analisar principalmente fatores do contexto. Os

Quadro 3

Fatores de promoção e de impedimento relativos aos processos de formulação de pesquisa e de política.

\begin{tabular}{ll}
\hline Fatores de promoção & Fatores de impedimento \\
\hline $\begin{array}{l}\text { Comunicação informal, relações informais, } \\
\text { relações pessoais }\end{array}$ & $\begin{array}{l}\text { Comunicação de resultados de pesquisa } \\
\text { apenas em revistas científicas }\end{array}$ \\
Equilíbrio entre os interesses de grupos & $\begin{array}{l}\text { Desinteresse dos pesquisadores em comunicarem } \\
\text { os resultados de pesquisa aos formuladores } \\
\text { de políticas }\end{array}$ \\
$\begin{array}{l}\text { Desenvolvimento e uso de canais formais } \\
\text { de comunicação }\end{array}$ &
\end{tabular}

\section{Quadro 4}

Fatores de promoção e impedimento relativos ao contexto político-institucional em que ocorre a pesquisa e a política.

\begin{tabular}{ll}
\hline Fatores de promoção & Fatores de impedimento \\
\hline Estabilidade política & Excessiva centralização do poder \\
$\begin{array}{l}\text { Pesquisadores com cargos de formuladores } \\
\text { de políticas }\end{array}$ & Gerenciamento hierárquico da informação \\
$\begin{array}{l}\text { Tamanho e homogeneidade da comunidade } \\
\text { científica }\end{array}$ & Descontinuidade administrativa \\
Urgência do problema & Restrições financeiras \\
\hline
\end{tabular}


resultados de Trostle et al. (1999) nos orientaram na definição de critérios para identificar os fatores promotores e de impedimento do uso de pesquisa na política considerando a realidade brasileira.

Os conceitos de pesquisa e política estabelecidos por Trostle et al. foram empregados no nosso estudo. Pesquisa foi definida como um processo estruturado de coleta, análise-síntese e interpretação exploratória ou descritiva de dados para responder uma questão teórica ou prática não visível nos dados existentes. Política foi considerada um guideline governamental ou organizacional que define princípios e prioridades para alocação de recursos.

Sintetizamos em um modelo lógico aspectos que consideramos relevantes da definição de componentes essenciais para utilização de pesquisa. Para Hartz (1999), um modelo teórico, também chamado de modelo lógico, procura identificar elos causais entre o que é feito no programa e os objetivos do mesmo.

Um modelo lógico descreve a seqüência de eventos, por meio de uma síntese dos componentes essenciais desenhados em um fluxo, demonstrando como o modelo teoricamente pode ser utilizado. Possui, como vantagem, a capacidade para resumir o funcionamento de um programa, ligando o processo aos resultados, a interação dos efeitos de seus componentes como impacto (Cosendy, 2000). Os elementos que compõem um modelo teórico podem variar, mas de modo geral incluem insumos (inputs), as atividades, os produtos (outputs) e os efeitos de curto, médio e longo prazo (Hartz et al. 1997).

Consideramos que o modelo teórico de utilização de pesquisa em políticas tentará incorporar elementos amplos e conexos que auxiliarão na resposta de perguntas-chave: quais aspectos do contexto explicam a utilização de pesquisa em políticas de saúde pública e quais fatores interferem na efetiva utilização de pesquisa em política?

Adotamos como elementos do modelo teórico: a) componentes-aspectos essenciais na utilização de pesquisa em políticas, b) objetivos de cada componente, c) produtos para cada objetivo, e) resultados intermediários e resultados finais do componente, em função do seu objetivo e produto.

Os componentes selecionados para o modelo teórico foram aqueles que, citados por Weiss (1979) e descobertos nos achados empíricos de Trostle et al. (1999), tiveram características singulares para explicar diferentes fato- res de contexto e de efetivação de utilização de pesquisa.

\section{Resultados e conclusões}

O modelo teórico de avaliação de um programa hipotético de fomento de pesquisas em saúde para utilização de resultados na formulação de políticas de saúde proposto pelas autoras é formado pelos seguintes componentes: a) Conteúdo de pesquisa; b) Comunicação; c) Interação; d) Atores; e) Pressão social e f) Financiamento (Figura 2).

Consideramos objetivo relevante no componente conteúdo de pesquisa a existência de um problema a ser solucionado com subsídios provenientes de evidências científicas; entretanto, faz-se necessária a clareza das conclusões e recomendações científicas para que os resultados possam ser disseminados e possam influenciar os tomadores de decisão.

No modelo, o resultado do conteúdo da pesquisa integra-se com o componente comunicação, já que neste, o elemento principal é a divulgação de informações científicas não apenas em canais formais de acesso restrito a pesquisadores, mas também, através de meios de comunicação informais que alcance atores com expressão política. Espera-se como resultado a formação de um conjunto de forças capazes de pressionar os tomadores de decisão a utilizarem subsídios científicos.

No componente interação é redimensionado o papel dos diferentes atores que devem participar de todo o processo, ou seja, desde a definição de problemas e seleção de temas prioritários, sendo que esta estratégia deve garantir demanda destes mesmos atores por acesso aos resultados obtidos em pesquisas.

Quanto maior for o equilíbrio de interesses, maior será a interatividade entre os atores. Quanto melhor for a compreensão pelos atores dos resultados de pesquisas, maior será a garantia de valorização de sua utilização.

A pressão social por políticas decorrentes dos componentes anteriores do modelo acarretaria a necessidade de transparência no processo de contratação de instituições e/ou pesquisadores que executem estudos de interesse público, o que contribuiria para a legitimação das decisões políticas subsidiadas por pesquisas.

Os componentes - conteúdo de pesquisa, comunicação, interação, atores e pressão social - determinam que o financiamento seja volta- 
Figura 2

Modelo teórico de utilização de pesquisa em política.

\begin{tabular}{|c|c|c|c|c|c|c|}
\hline \multirow[t]{2}{*}{ Componentes } & $\begin{array}{c}\text { Conteúdo da } \\
\text { pesquisa }\end{array}$ & Comunicação & Interação & Atores & Pressão social & Financiamento \\
\hline & $\downarrow$ & $\downarrow$ & $\downarrow$ & $\downarrow$ & $\downarrow$ & $\downarrow$ \\
\hline \multirow[t]{3}{*}{ Objetivos } & $\begin{array}{l}\text { Tema, } \\
\text { problema ou } \\
\text { necessidade }\end{array}$ & $\begin{array}{c}\text { Disseminação } \\
\text { de resultados } \\
\text { de pesquisas }\end{array}$ & $\begin{array}{l}\text { Definição de } \\
\text { problemas }\end{array}$ & $\begin{array}{c}\text { Interesses em } \\
\text { equilíbrio }\end{array}$ & $\begin{array}{c}\text { Pressão por } \\
\text { política }\end{array}$ & \begin{tabular}{|c|} 
Investimentos \\
em temas \\
prioritários
\end{tabular} \\
\hline & & $\begin{array}{l}\text { por canais } \\
\text { formais e } \\
\text { informais }\end{array}$ & em conjunto & & & \\
\hline & $\downarrow$ & $\downarrow$ & $\downarrow$ & $\downarrow$ & $\downarrow$ & $\downarrow$ \\
\hline \multirow[t]{2}{*}{ Produtos } & $\begin{array}{c}\text { Recomendações } \\
\text { específicas e } \\
\text { concretas }\end{array}$ & $\begin{array}{l}\text { Acesso dos } \\
\text { atores às } \\
\text { evidências }\end{array}$ & $\begin{array}{c}\text { Temas } \\
\text { prioritários } \\
\text { para pesquisa }\end{array}$ & $\begin{array}{c}\text { Compreensão } \\
\text { das evidências } \\
\text { científicas }\end{array}$ & $\begin{array}{c}\text { Desenvolvimen- } \\
\text { to de pesquisa } \\
\text { por agência ou }\end{array}$ & $\begin{array}{c}\text { Monitoramento } \\
\text { do desenvolvi- } \\
\text { mento de }\end{array}$ \\
\hline & $\downarrow$ & $\downarrow$ & $\downarrow$ & $\downarrow$ & $\downarrow$ & $\downarrow$ \\
\hline \multirow[t]{3}{*}{$\begin{array}{l}\text { Resultados } \\
\text { intermediários }\end{array}$} & $\begin{array}{l}\text { Disseminação } \\
\text { de resultados }\end{array}$ & $\begin{array}{l}\text { Pressão por } \\
\text { políticas }\end{array}$ & $\begin{array}{l}\text { Pressão por } \\
\text { resultados de } \\
\text { pesquisa }\end{array}$ & $\begin{array}{l}\text { Valorização da } \\
\text { aplicação dos } \\
\text { resultados de }\end{array}$ & $\begin{array}{c}\text { Legitimação } \\
\text { de decisão } \\
\text { política }\end{array}$ & $\begin{array}{l}\text { Resultado de } \\
\text { pesquisa par } \\
\text { resolver }\end{array}$ \\
\hline & & & & $\begin{array}{l}\text { pesquisa nas } \\
\text { políticas }\end{array}$ & & probiemas \\
\hline & $\downarrow$ & $\downarrow$ & $\downarrow$ & $\downarrow$ & $\downarrow$ & $\downarrow$ \\
\hline $\begin{array}{l}\text { Resultado final } \\
\text { ou impacto }\end{array}$ & \multicolumn{6}{|c|}{ Uso de pesquisa na formulação de políticas } \\
\hline
\end{tabular}

do para pesquisas de interesse da sociedade, monitoradas pelo controle social e resultem na solução de problemas atuais e previstos em cenários futuros.

Podemos afirmar que no modelo proposto os componentes conectam-se entre si.

Para avaliação de projetos de pesquisa a serem selecionados em licitações públicas, elaboramos uma matriz de critérios de efetividade (Quadro 5). As dimensões a serem incluídas são: a) estrutural - relativa ao conteúdo da pesqui- sa. Os indicadores propostos dizem respeito à consistência do referencial teórico, à pertinência dos objetivos em relação ao objeto de estudo; ao potencial de pesquisa responder a questões essenciais para a natureza dos problemas prioritários, à clareza da linguagem, à análise da viabilidade de aplicação concreta dos possíveis resultados e os custos, permitindo comparação entre projetos que concorram a um mesmo edital;

b) operacional - relativa a processos e atividades de pesquisa. Os indicadores dizem respeito 
à existência de tecnologia, custo de inovação tecnológica e motivação dos atores para exercerem pressão por implementação dos possíveis resultados;

c) estratégica - relativa a pesquisa e práticas de formulação de política. Os indicadores dizem respeito aos possíveis usos políticos dos resultados.

d) sistêmica - relativa aos fatores externos a pesquisa que influenciam a utilização de resultados nas políticas. Os indicadores dizem respeito à natureza do interesse político que gerou a demanda por pesquisa.

e) específica - relativa aos resultados e impactos. Os indicadores dizem respeito à geração de conflitos mínimos na formulação de políticas, contribuição que minimize possíveis riscos para saúde da população e a aceitabilidade em caso de mudanças de padrões sociais.

A matriz, para facilitar os cálculos, deverá alcançar um valor máximo múltiplo de 10 , por exemplo 1.000 pontos. Os critérios contidos nas dimensões deverão receber pontuação com base na valorização a ser dada pelos diversos atores que participarem da seleção. A pontuação final será resultante do somatório dos valores al- cançados em cada dimensão, que por sua vez resulta do somatório de pontos alcançado nos diferentes critérios/indicadores.

Para avaliar a viabilidade da utilização de resultados de pesquisas na formulação de políticas elaboramos matriz com critérios para identificação de fatores promoção e de impedimento (Quadro 6). A mensuração deverá ser baseada na descrição anterior para a matriz de efetividade, entretanto a resposta "sim" corresponderá a fatores de promoção e deverá receber pontuação positiva e a resposta "não" corresponderá a fatores de impedimento e deverá receber pontuação negativa. A soma algébrica dos diversos valores encontrados visará demonstrar o balanço de forças atuantes no processo pesquisa-política.

Finalmente, considerando a afirmativa de Trostle et al. (1999) de que inclusão de resultados de pesquisa em política é influenciada pelo contexto em que a mesma se desenvolve e que esse mesmo contexto é influenciado por ela, elaboramos a matriz de contexto (Quadro 7). Selecionamos as seguintes dimensões de contexto em que as pesquisas e as políticas ocorrem: eco-

Quadro 5

Matriz de efetividade na seleção de projetos de pesquisa.

\section{Dimensões}

Estrutural - conteúdo do projeto de pesquisa (critérios/indicadores)

- Qualidade do projeto

- Especificidade do projeto

- Linguagem do projeto

- Aplicação concreta

- Custo efetividade

Operacional - processos e atividades de pesquisa (critérios/indicadores)

- Requisitos técnicos para implementação dos resultados da pesquisa

- Nível de participação popular, dos usuários, para implementação dos resultados

- Custo para implementação dos resultados

Estratégica - pesquisa e práticas de formulação de política (critérios/indicadores)

- Utilização da pesquisa para reduzir incertezas

- Utilização da pesquisa para neutralizar oponentes

- Utilização da pesquisa para legitimar decisão política

Sistêmica - fatores externos a pesquisa (critérios/indicadores)

- Pesquisa encomendada para responder a pressões

- Pesquisa financiada para responder a aspectos oficiais

Específica - resultados e impactos na utilização de pesquisa (critérios/indicadores)

- Garantia de estabilidade na tomada de decisão

- Benefícios para a população

- Mudança de parâmetros sociais 
Quadro 6

Matriz de fatores de promoção e impedimento que influenciam a utilização de resultados de pesquisa.

Dimensões

Características dos resultados de pesquisa (critérios/indicadores)

- Os resultados da pesquisa têm consistência científica (teórica e metodológica)

- Há clareza de linguagem e apresentação sintética das recomendações da pesquisa

- Os resultados da pesquisa são específicos e concretos

Atores (critério/indicadores)

- Houve participação dos diferentes atores no monitoramento do desenvolvimento da pesquisa

- Há pressão de órgãos internacionais para utilização de evidências científicas referentes aos resultados de pesquisas sobre o tema

- Há forte influência e conexões das organizações oficiais de pesquisa sobre os formuladores de políticas sobre o tema

Processo de pesquisa e política (critérios/indicadores)

- Há espaço de articulação entre pesquisadores e formuladores de políticas

- Há canais de comunicação formais e informais para acesso dos diferentes atores aos resultados da pesquisa

- Há equilíbrio de interesses na comunidade científica considerando-se os resultados da pesquisa

Quadro 7

Matriz do contexto onde se desenvolve a construção de pesquisa e de política.

Dimensões

Econômica (critérios/indicadores)

- Há restrições financeiras para investimento em tecnologia para implantação dos resultados de pesquisa

- Há interesse de grupos financeiros nos resultados da pesquisa

- Há necessidade de recursos vultuosos para aplicação dos resultados da pesquisa

Política (critérios/indicadores)

- Há perspectiva de manejo político de forças que se oponham a utilização dos resultados da pesquisa

- Há demanda por resultados da pesquisa para aumentar pressão junto dos formuladores de política por sua utilização

- Há pesquisadores sobre o tema exercendo cargos de formuladores de políticas

Social (critérios/indicadores)

- Os resultados da pesquisa fornecem subsídios para a resolução de problema/necessidades sociais

- Os resultados da pesquisa permitem a formulação de política em reposta às pressões sociais

- Os resultados da pesquisa fornecem evidências para soluções inovadoras com a participação da sociedade

Cultural (critérios/indicadores)

- Os resultados da pesquisa são compatíveis com padrões sociais vigentes

- Há valorização pela sociedade dos resultados de pesquisa sobre o tema

- Há elevado background técnico dos formuladores de política e da mídia para compreensão de resultados de pesquisa sobre o tema

Técnico-gerencial (critérios /indicadores)

- Há influência da área de C\&T do Ministério da Saúde para formulação de políticas com base nos resultados da pesquisa

- Há apoio dos institutos de pesquisa para divulgação dos resultados de pesquisa junto de atores de expressão política

- Há oportunidades no âmbito político-administrativo para uso das informações científicas resultantes da pesquisa 
nômica, política, social, cultural e técnico-gerencial. Para mensuração propõe-se o mesmo método descrito para matriz de efetividade

$\mathrm{O}$ conjunto de ferramentas formado pelo modelo teórico e as matrizes configuram um guideline para operacionalização do processo de seleção de pesquisas e de avaliação da viabilidade de utilização de resultados na formulação de políticas.

A validação das matrizes deve ser realizada em oficinas com representantes de gestores, pesquisadores e formuladores de políticas. A finalidade é a revisão, a padronização de indicadores, a análise da viabilidade de coleta de da- dos ou a descoberta de novos componentes essenciais que não foram incluídos no modelo.

No decorrer desse trabalho procuramos sintetizar aspectos teóricos que propiciem a reflexão sobre o uso de resultados de pesquisa na tomada de decisão voltada para o bem-estar social. O modelo e matrizes propostos podem orientar a formulação de indicadores que permitam o monitoramento e avaliação da integração pesquisa e formulação de políticas, assim como contribuir para a construção de estratégias que venham garantir a utilização de evidências científicas como subsídio para formulação de políticas de saúde.

\section{Colaboradores}

FTS Elias trabalhou na revisão da literatura, análise e sistematização do artigo de Trostle et al., na elaboração do corpo do texto da introdução, metodologia e resultados, e na elaboração, em conjunto com a segunda autora, do modelo teórico e das matrizes com indicadores de avaliação. MAA Patroclo trabalhou na análise e sistematização do artigo de Carol Weiss, na revisão do corpo do texto da introdução, na metodologia e resultados, e na elaboração, em conjunto com a primeira autora, do modelo teórico e das matrizes com indicadores de avaliação. 


\section{Referências bibliográficas}

Academia Brasileira de Ciências (ABC) 1994. Encontros setoriais: contribuição de ciência e tecnologia para a área social em saúde, São Paulo.

Bartley M 1992. Authorities and partisans. The debate on unemployment and health. Edinburgh University Press, Edimburgo.

Brasil. Ministério da Saúde/Coordenação Geral de Desenvolvimento Científico e Tecnológico 1994. I Conferência Nacional de Ciência e Tecnologia em Saúde. Brasília.

Brownson RC, Newschaffer CJ \& Ali-Abi-Abarghoui F 1997. Policy research for disease prevention: challenges and practical recommendations. American Journal of Public Health 87:735-739.

COHRED 2000a. Working Group on Research to Action and Policy. Lessons in research to action and policy. Case studies from seven countries. Council on Health Research for Development (COHRED), Genebra.

Cosendy MAE 2000. Análise da implantação do programa de farmácia básica: um estudo multicêntrico em cinco Estados do Brasil. Tese de doutorado. ENSP/Fiocruz, Rio de Janeiro.

Davis P \& Howdwn-Chapman P 1996. Translating Research Findings into Health Policy. Soc. Sci Med. 43(5) 865-872.

Donnison D 1972. Research for policy. Minerva 10(4)519536.

Ferguson JH 1993. NIH consensus conferences: dissemination and impact. Ann. N Y Acad. Sciences 703:180-198.

Frenk J 1992. Balancing relevance and excellence: organizational responses to link research with decision making. Social Science and Medicine 35:1397-1404.

Hailey DM 1993. The influence of technology assessment by advisory bodies on health policy and practice. Health Policy 25:243-254.

Haines A \& Jones R 1994. Implementing findings of research. BMJ 308:1488-1492.

Hartz ZMA 1997. Avaliação em saúde: dos modelos conceituais à prática na análise de implantação de programas. Fiocruz, Rio de Janeiro.

Hartz ZMA 1999. Avaliação dos programas de saúde: perspectivas teórico-metodológicas e políticas institucionais. Ciência e Saúde Coletiva 4(2):341-353.

Mckinlay J 1993. The promotion of health through planned sociopolitical change: challenges for research and policy. Soc. Sci. Med. 36:109-117.

Natan C, Morrison A \& Stambaugh R 1975. The use of social science knowledge in policy decisions at the national level. Ann Arbor, Michigan: Institute for Social Research.
OPS 2000. La Reforma del Sector Salud en las Americas: Fortaleciendo los Vínculo entre Investigación y Políticas. Programa. Reunião de Montréal: Organización Panamericana de la Salud.

Pahl J 1992. Force for change or optional extra? The impact of research on policy in social work and social welfare, pp. 215-230. In Changing Social Work and Welfare. Open University Press, Milton Keynes.

Porter RW 1995. Knowledge utilization and the process of policy formation: toward a framework for Africa. Washington DC : Porter/Novelli for the SARA Project, Academy for Educational Development, January, UK.

Stocking B 1995 . Why research findings are not used by commissions and what can be done about it. Journal of Public Health Medicine 17:380-382.

Trostle J, Bronfmen M \& Langer A 1999. How do researchs influence decision-makers? Case studies of Mexicam policies. Health Policy and Planning 14(2):103-114.

Walt G 1994. How far does research influence policy? European Journal of Public Health 4:233-235.

Weiss CH 1975. Evaluation research in the political context, pp. 13-25. In EL Struening \& M Guttentag (eds.). Handbook of evaluation research. Sage, Londres.

Weiss CH 1978. Improving the linkage between social research and public policy, pp. 22-81. In LE Lynn (ed.). Knowledge and policy: the uncertain connection. National Academy of science, Washington DC.

Weiss CH 1979. The many meanings of research utilization. Puplic Administration Review September/October, 426 a 431.

Weiss CH 1991. Policy research: data, ideas, or arguments?, pp. 307-332. In P Wagner et al. (eds.). Social sciences and modern states, national experiences and theoretical crossroads. Cambridge University Press, Cambridge.

Weiss J 1976. Using social science for social policy. Policy Studies Journal 4(3):236.

WHO/ACHR 1998. Investing in health research and development. Genebra.

WHO Comittee on Health Research 1998. A Research Agenda for Science and Technology to Support Global Health Development. WHO/OMS - Collaborating Venture for Global Modeling of Health. 1998.

Wittrock B 1991. Social knowledge and public policy: eight models of interaction. In P Wagner et al. Social sciences and modern states. national experiences and theoretical crossroads. Cambridge University Press, Cambridge.

Artigo apresentado em 14/04/2004

Aprovado em 27/07/2004

Versão final apresentada em 20/11/2004 